Pacific Journal of Mathematics

CLASS NUMBERS OF IMAGINARY CYCLIC QUARTIC FIELDS 


\title{
CLASS NUMBERS OF IMAGINARY CYCLIC QUARTIC FIELDS AND RELATED QUATERNARY SYSTEMS
}

\author{
RICHARD H. HUDSON
}

A proof is given of an explicit Dirichlet-type class number formula for imaginary cyclic quartic fields obtained in 1980 by Hudson and Williams and, in a slightly different form, by Setzer. The HudsonWilliams formula is used to study the solvability of the quaternary quadratic form

$$
\begin{aligned}
16 p^{k} & =x^{2}+2 q u^{2}+2 q v^{2}+q w^{2}, \\
x w & =a v^{2}-2 b u v-a u^{2}, \quad(x, u, v, w, p)=1
\end{aligned}
$$

for exponents $k \geq 1$. Included is a table from which every class number $h(k)$ of the quartic field $k=Q(i \sqrt{2 q+2 a \sqrt{q}}), q \equiv 5(\bmod 8)$ a prime, may be determined for $q<10000$. Finally, a quartic analog of the well-known result that the number of quadratic residues in $(0, p / 2)$ exceeds the number in $(p / 2, p)$ if $p \equiv 3(\bmod 4)$ is proven using one of Dirichlet's less well-known class number formulas.

1. Introduction. Explicit Dirichlet-type class number formulas for imaginary cyclic quartic fields were obtained in 1980 by Hudson and Williams and independently by Setzer. In this paper we sketch in $\$ 2$ the proof of the Hudson-Williams formula and show that these two formulas are easy consequences of one another. However, the Hudson-Williams formulation is particularly useful for studying the solvability of the quaternary quadratic form

$$
\begin{aligned}
& 16 p^{k}=x^{2}+2 q u^{2}+2 q v^{2}+q w^{2}, \\
& x w=a v^{2}-2 b u v-a u^{2}, \quad(x, u, v, w, p)=1,
\end{aligned}
$$

for $k \geq 1$. We show in $\S \S 3-5$ that solvability of this form depends heavily on the relative class number $h^{*}=h(k) / h(Q(\sqrt{q}))$ of the imaginary cyclic quartic field

$$
K=Q(i \sqrt{2 q+2 a \sqrt{q}})=Q(i \sqrt{2 q-2 a \sqrt{q}})
$$

where $q \equiv 5(\bmod 8)=a^{2}+b^{2}(a$ odd, $b>0)$ will denote a prime $>5$ throughout and $h(Q(\sqrt{q}))$ the class number of the unique quadratic subfield $Q(\sqrt{q})$ of $K$. 
Recall the well-known result that the number of quadratic residues in the interval $(0, p / 2)$ exceeds the number in the interval $(p / 2, p)$ if $p \equiv 3$ (mod 4). Using a class number formula of Dirichlet we prove an analogous result for quartic residues in $\S 6$ for primes $q \equiv 5(\bmod 8)$.

Finally, in $\$ 7$, we enclose a table of values of $h^{*}$ for every $q<10000$. These were computed on two different home computers and cross-checked for accuracy.

2. The Hudson-Williams formula. Let $N_{0}, N_{1}, N_{2}, N_{3}$ denote the number of quartic residues in the intervals $(0, p / 4),(p / 4, p / 2)$, $(p / 2,3 p / 4),(3 p / 4, p)$, respectively. Bennett Setzer [16] proved that

$$
h^{*}=h(k) / h(Q(\sqrt{q}))=\frac{1}{2}\left(\left(N_{3}-N_{0}\right)^{2}+\left(N_{2}-N_{1}\right)^{2}\right) .
$$

Let $\chi_{1}$ denote the nonprincipal character $(\bmod q)$ of order 4 such that $\chi_{1}(2)=+i$ and let $c_{1}, c_{2}, c_{3}$ denote the cosets which may be formed with respect to the subgroup of fourth powers $(\bmod q)$ which we denote by $c_{0}$. Define the coset sums $S_{0}, S_{1}, S_{2}, S_{3}$ by

$$
S_{J}=S_{J}\left(\chi_{1}\right)=\frac{1}{q} \sum_{n=i^{\prime}}^{q-1}, \quad j=0,1,2,3 .
$$

Hudson and Williams [11] proved that

$$
h^{*}=\frac{1}{5}\left(\left(S_{2}-S_{0}\right)^{2}+\left(S_{3}-S_{1}\right)^{2}\right) .
$$

The formulation (2.3), announced by Hudson at the A.M.S. meeting in Ann Arbor in 1980, is more convenient to use than (2.1) in investigating solutions of the quaternary quadratic form

$$
\begin{aligned}
& 16 p^{k}=x^{2}+2 q u^{2}+2 q v^{2}+q w^{2}, \\
& x w=a v^{2}-2 b u v-a u^{2}, \quad(x, u, v, w, p)=1 .
\end{aligned}
$$

The form (2.4) has been studied by, among others, Dickson [6], Lehmer [12], Whiteman [17], Muskat and Zee [13], and Hudson, Williams, and Buell [10].

Throughout the paper we let

$$
f=\max \left\{\left|S_{0}-S_{3}\right|,\left|S_{1}-S_{2}\right|\right\} .
$$

Hudson and Williams [10, §4] proved that (2.4) is always solvable when $k=f$ for primes $p \equiv 1(\bmod q)$. Although authors to-date have dealt exclusively with primes $p \equiv 1(\bmod q)$, Hudson and Buell [4] noticed that 
this restriction is artificial, as existence (or non-existence) of solutions of (2.4) depends only on the condition that $p=q f+r$, where $r$ is any quartic residue of $q$. It would be highly desirable to have a proof analogous to that in $\S 4$ of $[10]$ when $(r / q)_{4}=+1, r \neq 1$ (see remark following Theorem 4.1).

We now sketch the proof of (2.3). Since the degree of $K$ over the rational field $Q$ is $4, K$ is an Abelian extension of $Q$. Hence the class number $h(K)$ is given by (see, e.g. [14, p. 372]),

$$
h(K)=\frac{w(K)|d(K)|^{1 / 2}}{2^{r_{1}+r_{2}} \pi^{r_{2}} R(K)} \prod_{\chi} L\left(1, \chi^{\prime}\right) .
$$

Appealing to Edgar and Peterson [8] it is easy to see that all the units of $K$ are given by $\pm \varepsilon^{k}(k=0,1, \ldots)$ where $\varepsilon$ is the fundamental unit ( $>1$ ) of $Q(\sqrt{q})$ and, consequently, to deduce that $w(K)=2$ (as $q>5$ ) and $R(K)=2 \log \varepsilon$.

Appealing to the work of Adrian Albert [1] we obtain that $d(K)=q^{3}$ (see, in particular, equations 9, 34-37 and Theorem 10 of [1]).

Let $\zeta_{q}=e^{2 \pi i / q}$. It is not difficult to show (see Hasse [9]) that the nonprincipal characters $\chi(\bmod q)$ which are trivial on the subgroup $c_{0}$ (the fourth powers $(\bmod q)$ ) are precisely $\chi_{1}$ and $\chi_{3}$, the two nonprincipal characters $(\bmod q)$ of order 4 , and the Legendre symbol $(n / q)$. For convenience we distinguish $\chi_{1}$ from $\chi_{3}$ by choosing $\chi_{1}(2)=i, \chi_{3}(2)=-i$ so that 2 belongs to $c_{1}$ throughout the paper.

Let $\chi^{\prime}$ be the primitive character induced by $\chi$ and note that $\chi^{\prime}=\chi$ since $q$ is prime. Hence

$$
\prod_{\chi} L\left(1, \chi^{\prime}\right)=L\left(1, \chi_{1}\right) L\left(1, \chi_{2}\right) L\left(1, \chi_{3}\right)
$$

Appealing to Berndt [2, Th. 3.2] we obtain (as $\chi_{1}$ is an odd character) that

$$
\text { (2.7) } L\left(1, \chi_{1}\right) L\left(1, \chi_{3}\right)=\pi^{2} \frac{\sum_{0<n<q / 2} \chi_{1}(n) \sum_{0<n<q / 2} \chi_{3}(n)}{-G\left(\chi_{1}\right) G\left(\chi_{3}\right)\left(\chi_{1}(2)-2\right)\left(\chi_{3}(2)-2\right)}
$$

where

$$
G(\chi)=\sum_{j=1}^{q-1} \chi(j) \zeta_{q}^{j}
$$


Since $G\left(\chi_{3}\right)=\overline{-G\left(\chi_{1}\right)}$ we have $-G\left(\chi_{1}\right) G\left(\chi_{3}\right)=q$ and, moreover, $\left(\chi_{1}(2)-2\right)\left(\chi_{3}(2)-2\right)=5$ so $(2.7)$ becomes

$$
L\left(1, \chi_{1}\right) L\left(1, \chi_{3}\right)=\frac{\pi^{2}}{5 q} \sum_{0<n<q / 2} \chi_{1}(n) \sum_{0<n<q / 2} \chi_{3}(n) .
$$

From Dirichlet's class number formula [7] we have

$$
L\left(1, \chi_{2}\right)=\sum_{n=1}^{\infty}\left(\frac{n}{q}\right) / n=\frac{2 \log \varepsilon}{\sqrt{q}} h(Q(\sqrt{q}))
$$

and using an easy generalization of an identity of Cauchy [5] given in [11] we obtain

$$
\sum_{0<n<q / 2} \chi(n)=(2+\chi(2))\left(\left(S_{2}-S_{0}\right)+\left(S_{3}-S_{1}\right) i\right) .
$$

It now follows at once from (2.1)-(2.4) that

$$
h^{*}=\frac{1}{5}\left(\left(S_{2}-S_{0}\right)^{2}+\left(S_{3}-S_{1}\right)^{2}\right),
$$

proving (2.3).

REMARK. The above proof is given here both for completeness and in the hope that this proof will be helpful to a future author in deriving an analogous formula for real cyclic quartic fields.

3. Solutions of (1.1) when $h^{*}$ is a perfect square. As mentioned in the introduction, the author and Kenneth Williams opted not to publish the class number formula (2.3) after jointly deriving the following formulas relating the coset sums $S_{j}$ to the numbers of quartic residues $N_{i}$, $i=0,1,2,3$, in subintervals of $p$. Such formulas are easily derivable in the quadratic and quartic cases and may well have higher power analogues.

Theorem 3.1. For $S_{j}$ defined as in (2.2), $j=0,1,2,3$, we have the following formulas.

$$
\begin{aligned}
& S_{0}=\frac{1}{2}\left(\frac{q-1}{4}-\frac{3}{5}\left(N_{0}-N_{3}\right)-\frac{1}{5}\left(N_{1}-N_{2}\right)\right), \\
& S_{1}=\frac{1}{2}\left(\frac{q-1}{4}-\frac{1}{5}\left(N_{0}-N_{3}\right)+\frac{3}{5}\left(N_{1}-N_{2}\right)\right), \\
& S_{2}=\frac{1}{2}\left(\frac{q-1}{4}+\frac{3}{5}\left(N_{0}-N_{3}\right)+\frac{1}{5}\left(N_{1}-N_{2}\right)\right), \\
& S_{3}=\frac{1}{2}\left(\frac{q-1}{4}+\frac{1}{5}\left(N_{0}-N_{3}\right)-\frac{3}{5}\left(N_{1}-N_{2}\right)\right) .
\end{aligned}
$$


Proof. Since (3.1) may be reformulated as

$$
\begin{aligned}
& \frac{-3}{10}\left(N_{0}-N_{3}\right)-\frac{1}{10}\left(N_{1}-N_{2}\right)=S_{0}-\frac{q-1}{8}, \\
& \frac{3}{10}\left(N_{0}-N_{3}\right)+\frac{1}{10}\left(N_{1}-N_{2}\right)=S_{2}-\frac{q-1}{8}, \\
& \frac{-1}{10}\left(N_{0}-N_{3}\right)+\frac{3}{10}\left(N_{1}-N_{2}\right)=S_{1}-\frac{q-1}{8}, \\
& \frac{1}{10}\left(N_{0}-N_{3}\right)-\frac{3}{10}\left(N_{1}-N_{2}\right)=S_{3}-\frac{q-1}{8},
\end{aligned}
$$

we have, after subtracting the second equation from the first and the fourth from the third above, and squaring, that

$$
\frac{9}{100}\left(N_{0}-N_{3}\right)^{2}+\frac{1}{100}\left(N_{1}-N_{2}\right)^{2}=\left(S_{0}-S_{2}\right)^{2}
$$

and

$$
\frac{1}{100}\left(N_{0}-N_{3}\right)^{2}+\frac{9}{100}\left(N_{1}-N_{2}\right)^{2}=\left(S_{1}-S_{3}\right)^{2} .
$$

Upon adding we obtain

$$
\frac{1}{2}\left(\left(N_{0}-N_{3}\right)^{2}+\left(N_{1}-N_{2}\right)^{2}\right)=\frac{1}{5}\left(\left(S_{0}-S_{2}\right)^{2}+\left(S_{1}-S_{3}\right)^{2}\right) .
$$

These are equal by the formula of Setzer (2.1) and the formula of Hudson-Williams (2.3), completing the proof of Theorem 3.1.

Solutions to the Diophantine system (1.1) fall into two distinct cases (Cases A and B in [10]). Throughout this section we assume we are in the former case so that

$$
f=\left|S_{0}-S_{2}\right|=\left|S_{1}-S_{2}\right| \text {. }
$$

It follows from (2.3) that $h^{*}$ is a perfect square. The converse is not true as evidenced by the data (see Tables in $\S 7)$ for $q=181\left(S_{0}=26, S_{1}=22\right.$, $\left.S_{2}=19, S_{3}=23 ; h^{*}=25\right)$. We therefore begin by noting a simple condition that (3.2) holds for all $q$ when $h^{*}$ is a perfect square.

LEMMA 3.1. Let $h^{*}$ be a perfect square. Then $f=\left|S_{0}-S_{2}\right|=\left|S_{1}-S_{3}\right|$ for all $q$ provided $h^{*}$ has no prime factor $\equiv 1(\bmod 4)$.

Proof. In view of (2.3) it is an immediate consequence of elementary number theory that $5 h^{*}$ can be expressed as the sum of two squares in exactly one way since $h^{*}$ has no prime factor of form $4 n+1$ by assumption. Thus

$$
h^{*}=\left|S_{0}-S_{2}\right|^{2}=\left|S_{1}-S_{3}\right|^{2}=f^{2} \text {. }
$$


We note in passing that if $5 h^{*}$ contains $S$ more prime factors of form $4 n+1$ than of form $4 n+3$ so that $5 h^{*}$ can be expressed as the sum of two squares in $S$ ways, then there generally exist $q$ for which each of the $S$ possibilities occurs. For example, for $5 h^{*}=5 \cdot 289=5 \cdot 17 \cdot 17=22^{2}+$ $31^{2}=34^{2}+17^{2}=38^{2}+1^{2}$, we have

$\begin{array}{lcc}\underline{q} & \frac{\left|N_{0}-N_{3}\right|}{2797} & \frac{\left|N_{1}-N_{2}\right|}{31} \\ 3581 & 32 & 17 \\ 9293 & 38 & 1\end{array}$

In order to understand how these $S$ possibilities arise in terms of the $N_{i}, i=0, \ldots, 3$, we need to prove the following theorem.

THEOREM 3.2. Let $q$ be a prime with $f=\left|S_{0}-S_{2}\right|=\left|S_{1}-S_{3}\right|$. Then we have

$$
h^{*}=\left|N_{3}-N_{0}\right|=f, \quad\left|N_{1}-N_{2}\right|=2 f,
$$

or

$$
h^{*}=\left|N_{1}-N_{2}\right|=f, \quad\left|N_{0}-N_{3}\right|=2 f .
$$

Proof. We need only prove, in view of Lemma 3.1, that

$$
h^{*}=\left|\left(N_{3}-N_{0}\right)-\left(N_{2}-N_{1}\right)\right| \text {. }
$$

To do this we use (3.1) assuming, w.l.o.g., that $\left(h^{*}\right)^{1 / 2}=S_{2}-S_{0}$. Then from (3.1) we have

$$
\begin{aligned}
& 5\left(S_{2}-S_{0}\right)=3 N_{0}+N_{1}-N_{2}-3 N_{3}, \\
& 5\left(S_{3}-S_{1}\right)=-N_{0}+3 N_{1}-3 N_{2}+N_{3},
\end{aligned}
$$

so that $4 N_{0}-2 N_{1}+2 N_{2}-4 N_{3}=0$ since $\left|S_{2}-S_{0}\right|=\left|S_{3}-S_{1}\right|$ by hypothesis. Upon addition we obtain

$$
-5 N_{0}+5 N_{1}-5 N_{2}+5 N_{3}=5\left(S_{3}-S_{1}\right),
$$

so that

$$
\left|\left(N_{3}-N_{0}\right)-\left(N_{2}-N_{1}\right)\right|=\left|S_{3}-S_{1}\right|=f .
$$

If $\left(h^{*}\right)^{1 / 2}=S_{0}-S_{2}, S_{3}-S_{1}$, or $S_{1}-S_{3}$ the proof is obtained similarly and may be omitted.

It follows from (4.20) of [10] that (1.1) is solvable for the exponent $k=f$ in view of (3.2). It may also be solvable for $k=1$ depending on how 
$p$ splits as a prime ideal but this is rarely the case for small $p$ unless, of course, $f=1$. Data suggests that Theorem 3.2 has the consequence that (1.1) is solvable only for exponents $k$ which are multiples of $f$ with exactly $\alpha$ solutions for each multiple $\alpha f$ unless $p$ is an "exceptional" prime such that (1.1) is solvable when $k=1$ even though $f>1$. If $q \leq 61$ there are exactly $k$ solutions for each exponent $k$ (see [10, §6], [4], [16]).

EXAMPLE. Let $q=149$. Using the generating techniques developed in [4] one can illustrate the above remarks for $p=1193 \equiv 1(\bmod q)$. However one can find and check solutions if $p$ is small without using vast amounts of computer time, so we choose to illustrate the above (see Remark in \$4) for $p=5$ noting $(5 / 101)_{4}=+1$. Direct computation for $(q, p)=(101,5)$ gives the following solutions $(x, u, v, w)$ of $(1.1)$ :

$\begin{array}{ll}\text { Exponent } & \text { Solution(s) } \\ \neq=3 \alpha, \alpha \leq 11 & \text { NONE } \\ k=3 & (19,1,2,1) \\ k=6 & (53,3,20,29),(245,4,21,19) \\ k=9 & (289,14,115,427),(3287,43,254,67), \\ & (4032,120,168,124) .\end{array}$

4. Solvability of (1.1) when $h^{*}=5,13$, and 17 . For $q=109$ with $h^{*}=17$ and $p=3,5$, and 7 , the system (1.1) is solvable for the seemingly random sequence of exponents $5,8,10,11,13,14,15, \ldots$ In this section we show exactly which exponent (1.1) is solvable for when it is not solvable for $k=1, h^{*} \leq 17$ and $\left|S_{0}-S_{3}\right| \neq\left|S_{1}-S_{2}\right|$.

It is easy to see that $h^{*} \equiv 1(\bmod 4)$ since $S_{0}+S_{2}=S_{1}+S_{3}=$ $(q-1) / 4 \equiv 1(\bmod 2)$ as $q \equiv 5(\bmod 8)$ so that

$$
\frac{1}{5}\left(\left(S_{2}-S_{0}\right)^{2}+\left(S_{3}-S_{1}\right)^{2}\right) \equiv 1(\bmod 4) .
$$

It may be worth noting from Table $1, \S 7$, the values of $q<10,000$ for which $h^{*}=1,5,9,13$, and 17:

$$
\begin{aligned}
& h^{*}=1: q=5,13,29,37,53,61, \\
& h^{*}=5: q=101,157,173,197,349,373, \\
& h^{*}=9: q=149,293,661, \\
& h^{*}=13: q=269,317,397,509,557,1789, \\
& h^{*}=17: q=109,229,227,821,853 .
\end{aligned}
$$


Quartic class numbers grow rapidly so that it is unlikely that the above list does not include all such $q$. Setzer [16] has proved that $q \leq 61$ when $h^{*}=1$ and it should be possible to modify his elegant argument to obtain upper bounds for $q$ for larger values of $h^{*}$.

For $q=101, p=607$, we have $f=3$ and (1.1) is solvable for $k=3$ with $(x, u, v, w)=(-8185,-966,1971,-5013)$. One would anticipate from $\S 3$ that (1.1) would be solvable for the exponent $k=6$ and have two solutions. However, determination of exponents for which (1.1) is solvable is far more complicated when $\left|S_{0}-S_{2}\right| \neq\left|S_{1}-S_{3}\right|$. For reasons developed in $\S 4$ the "missing" second solution for $k=6$ is, in fact, a solution for the exponent $k=4$, namely $(x, u, v, w)=(1017773,-11298,72615$, 21177). This is obtained by generating an imprimitive "solution" of (1.1) for $k=6$ (that is, one which fails to be a solution only since $(x, u, v, w, p) \neq 1)$ and then dividing each of $x, u, v$, and $w$ by $p$. The legitimacy of this procedure is rooted in Theorem 4.1 of [4] and the deep properties (5.41)-(5.43) of [10] which show that

$$
p^{\left(S_{n}-S_{m}\right)}\left\|\left(x^{2}-q w^{2}\right), \quad p^{\left(S_{n}-S_{m}\right)}\right\|(b x w+q u v),
$$

where $S_{m}$ is the smallest and $S_{n}$ the next smallest coset sum.

We consider now the typical case of a pair $(q, p)$ with $h^{*}>1$ and $S_{n}-S_{m}=1$ for which (1.1) is not solvable for any exponent $k$ less than

$$
f=\max \left\{\left|S_{0}-S_{2}\right|,\left|S_{1}-S_{3}\right|\right\}
$$

but is solvable for $k=2 f-2$. Given these conditions we now show that (1.1) is solvable for every $k \geq 3$ if $h^{*}=5$ and for the exponents 5, 8, 10, 11 , and every exponent greater than 12 if $h^{*}=13$ or 17 .

THEOREM 4.1. If $h^{*}=1$ then (1.1) is solvable for every $k>1$. If $h^{*}=1$ then (1.1) is solvable for every $k \geq 1$ iff it is solvable for $k=1$. If $h^{*}=5$ and (1.1) is not solvable for $k<f$ then it is insolvable for at most 2 values of $k(k=1,2)$. If $h^{*}=13$ or 17 and (1.1) is not solvable for $k<f$ then it is insolvable for at most 8 values of $k(k=1,2,3,4,5,7,9$, and 12).

Proof. The Theorem follows immediately from [10] and [4, Th. 4.1] noting simply that solutions for $k=m$ where $m \leq f$ and for $k=2 m-2$ (which exist in view of [10]) yield solutions (see Example below) for $k=2 m, 3 m-4,3 m-2,3 m, 4 m-6,4 m-4,4 m-2,4 m$, etc., and $\alpha m-(2 \alpha-2)<(\alpha-1) m$ for $\alpha \geq 3$ if $h^{*}=5$ as we then have $f=3$ and for $\alpha \geq 4$ if $h^{*}=13$ or 17 as then $f=5$. 
REMARK. Since the proof of solvability of (1.1) for $k=f$ rests on the assumption that $p \equiv 1(\bmod q)$ the above argument is not valid for $p=$ $q f+r,(r / q)_{4}=+1$; however, data proved by Duncan A. Buell favors the truth of all theorems presented in $\$ \S 3-5$ for all such quartic residues $r$.

We now show how to use Theorem 4.1 to actually generate the explicit solutions of $(1.1)$ for $(q, p)=(109,3)$.

EXAMPLE. Let $q=109$ and let $p=3$. With the signs of $a$ and $b$ chosen so that $a=-3, b=10$, it is easy to see that $(x, u, v, w)=(10,-2,2,4)$ is a solution of (1.5) for $k=5$. Computer data shows that there are no solutions for $k=1,2,3,4,5,7,9$ and 12 . The solution for $k=8$ arises as follows.

Using Theorem 4.1 of [4], noting that $S_{n}-S_{m}=1$ (see Table 2), so that by (5.4.1)-(5.4.3) of [10] the imprimitive "solution" of (1.5) for $k=10$ has each of $x, u, v, w$ divisible by $p$ (and so $x^{2}+2 q\left(u^{2}+v^{2}\right)+q w^{2}$ divisible by $p^{2}$ ) we obtain, applying (4.2) and (4.3) of [4], that

$$
\begin{gathered}
x_{8}=\frac{x^{2}-q w^{2}}{4 p}=112, \quad u_{8}=\frac{x u-a u w+a v w+x v}{4 p}=-4, \\
v_{8}=\frac{-x u+b v w-b u w-a u w+a v w+x v}{4 p}=20, \\
w_{8}=-\frac{1}{2}\left(b v^{2}+2 a u v-b u^{2}\right)=-4 .
\end{gathered}
$$

It is easily checked that $\left(x_{8}, u_{8}, v_{8}, w_{8}\right)=(-112,-4,+20,-4)$ is indeed a solution of (1.1) for $k=8$. The solution for $k=10$ is obtained from the solutions for exponents 5 and 8 by applying (4.2) and (4.3) of [4] and dividing by $p$ as for $k=8$. In this way the author has generated solutions for exponents up to 17 and checked these against direct computer data.

5. Solvability of (1.1) in the general case. We begin this section by proving the following theorem.

THEOREM 5.1. The system (1.1) is solvable for every sufficiently large exponent $k$ provided $h^{*}=1$ or $\left|S_{0}-S_{3}\right| \neq\left|S_{1}-S_{2}\right|$ and the smallest exponent $m$ that (1.1) is solvable for is odd.

Proof. It follows from [10, §6] and Theorem 4.1 of [4] that (1.1) is solvable for every $k$ if $h^{*}=1$. If on the other hand $\left|S_{0}-S_{3}\right| \neq\left|S_{1}-S_{2}\right|$ and $m$ is the smallest exponent that (1.1) is solvable for, then by Theorem 
4.1 of [4], (1.1) is solvable for the exponents $k=m, 2 m-2\left(S_{n}-S_{m}\right)$, $2 m, 3 m-4\left(S_{n}-S_{m}\right), 3 m-2\left(S_{n}-S_{m}\right), 3 m, 4 m-6\left(S_{n}-S_{m}\right)$, etc., that is for each $\alpha \geq 1$ for the exponents $k=\alpha m-(2 \alpha-2)\left(S_{n}-S_{m}\right)$, $\alpha m-(2 \alpha-4)\left(S_{n}-S_{m}\right), \alpha m-(2 \alpha-6)\left(S_{n}-S_{m}\right), \ldots, \alpha_{m}-$ $2\left(S_{n}-S_{m}\right), \alpha m$, where the products in parentheses are interpreted to be zero if they are not positive. Each of these expressions is congruent to $m$ modulo $2\left(S_{n}-S_{m}\right)$. Since $m$ is assumed odd and $2\left(S_{n}-S_{m}\right)$ is even the terms $\alpha m$ run through a complete residue class modulo $2\left(S_{n}-S_{m}\right)$ as $\alpha$ runs through any $2\left(S_{n}-S_{m}\right)$ consecutive integers. Thus an upper bound (although not best possible) for the exponent $b$ such that (1.1) is solvable for all $k>b$ is provided by determining the value of $\alpha$ such that

$$
\alpha m-(\alpha-1)\left(2 S_{n}-2 S_{m}\right) \leq\left(\alpha-2\left(S_{n}-S_{m}\right)\right)(m) .
$$

Since (5.1) clearly holds for $\alpha \geq m+1$, (1.1) is solvable for every exponent $k \geq m^{2}-\left(S_{n}-S_{m}\right)(m)$.

EXAMPLE. Let $q=181, p=7$, so that $h^{*}=25$. For $p \equiv 1(\bmod q)$ Hudson and Williams [10] have proved that (1.1) is solvable for $f=7$ (see Table 2) and direct computation shows that this is the smallest exponent for which (1.1) is solvable for the pair $(181,7)$. Since $S_{m}=22$ and $S_{n}=23$, Theorem 5.1 asserts that (1.1) is solvable for every $k \geq 42$. This is, we note, not a best possible bound although we also note that there is an exponent greater than $(m-1)^{2}-2\left(S_{n}-S_{m}\right)(m-1)=30$ for which a solution to (1.1) cannot be generated via Theorem 4.1 of [4] so it may not be possible to improve the bound greatly.

COROLlaRY. If for a pair $(q, p)$ the system (1.1) is solvable for $k \leq 2\left(S_{n}-S_{m}\right)+1$ then it is solvable for every $k \geq 4\left(S_{n}-S_{m}\right)+2$.

Proof. The theorem is immediate from the last sentence of the proof of Theorem 5.1.

EXAmple. Let $q=181, p=5$. Computer data provided by Duncan Buell shows that $(1.1)$ is solvable for $(q, p)=(181,5)$ when $k=3$. (The solution is $(x, u, v, w)=(3,0,1,3))$. Since $S_{n}-S_{m}=23-22=1,3=$ $2\left(S_{n}-S_{m}\right)+1$, and it follows from the above corollary that (1.1) is solvable for this pair for every $k \leq 4\left(S_{n}-S_{m}\right)+2=6$ (in contrast to the previous example). In fact as it is easily seen to be solvable for $k=4$ and 5 with $(x, u, v, w)=(81,0,3,1)$ in the former case and $(15,4,9,9)$ in the latter so that $k=1$ and 2 are the only exponents for which (1.5) is 
insolvable. In general if (1.1) is solvable for $k<f=\max \left\{\left|S_{0}-S_{2}\right|\right.$, $\left.\left|S_{1}-S_{3}\right|\right\}$ the system is solvable for exponents one would expect for a smaller value of $h^{*}$ (in this case the exponents one would expect when $h^{*}=5$ : see $\S 4$ ).

REMARKs. The results in this section are unsatisfactory in two major aspects. First, the results (4.20) and (5.41)-(5.43) of [10] have only been proved for $p \equiv 1(\bmod q)$ although they appear to hold for $p=q f+r$, $(r / q)_{4}=+1$. The generating technique given in Theorem 4.1 of [4] does not depend on the assumption that $r=1$. It would be highly desirable to have a proof in the general case (such a proof would require Brewer sums and Stickelberger's theorem). Second, the bound in Theorem 5.1 is not best possible and a more precise bound (holding for all $q$ ) would be desirable.

6. A consequence of a class number formula of Dirichlet. I close this paper with a theorem on the distribution of quartic residues in the subintervals $(0, q / 4),(q / 4, q / 2),(q / 2,3 q / 4),(3 q / 4, q)$ (see the last two columns of Table 2$)$.

For every prime $q \equiv 5(\bmod 8)$ it is the case that

$$
N_{0}+N_{3}>N_{1}+N_{2} \text {. }
$$

Proof. Let $c_{0}, c_{1}, c_{2}, c_{3}$ be defined as before, let $R_{1}$ and $T_{1}$ denote the numbers of quadratic residues and quadratic nonresidues respectively in $(0, q / 4)$, and let $R_{2}$ and $T_{2}$ denote the numbers of quadratic residues and quadratic nonresidues respectively in $(q / 4, q / 2)$. Then $N_{0}+N_{3}$ is the number of elements of $c_{0}$ in $(0, q / 4)$ plus the number in $(3 q / 4, q)$. But $N_{3}$ is clearly also the number of elements of $c_{2}$ in $(0, q / 4)$ as $q \equiv 5(\bmod 8)$ rather than $\equiv 1(\bmod 8)$. Thus $N_{0}+N_{3}=R_{1}$. Similarly $N_{1}+N_{2}=R_{2}$.

As $q \equiv 1(\bmod 4)$ we have trivially

$$
\sum_{0<n<q / 2}\left(\frac{n}{q}\right)=0
$$

so that

$$
\sum_{q / 4<n<q / 2}\left(\frac{n}{q}\right)<0
$$

in view of (1.2) of [2] (proved first by Dirichlet). It follows that $R_{2}<T_{2}$. Assume now that $N_{0}+N_{3}<N_{1}+N_{2}$, that is $R_{1}<R_{2}$. As $T_{2}=R_{1}$ we must have $R_{1}<R_{2}<T_{2}=R_{1}$, an obvious contradiction. This completes the proof. 
REMARK. Let $u_{0}, u_{1}, u_{2}, u_{3}$ denote the numbers of integers in the subintervals $(0, q / 4),(q / 4, q / 2),(q / 2,3 q / 4),(3 q / 4, q)$, respectively, which are quadratic residues but not quartic residues $(\bmod q)$. It is clear from the proof of Theorem 6.1 that we have, in addition, that for all $q \equiv 5$ $(\bmod 8)$,

$$
u_{0}+u_{3}>u_{1}+u_{2} \text {. }
$$

\section{TABLE 1}

Class Numbers of $h^{*}(K)=h(K) / h(Q(\sqrt{q}))$ for $5 \leq q<10,000$

\begin{tabular}{|c|c|c|c|c|c|c|c|c|c|c|c|c|c|c|c|c|c|}
\hline$q$ & $\mathbf{h}^{*}$ & $q$ & $h^{*}$ & 9 & $\mathbf{h}$ & $q$ & $\mathbf{h}^{\star}$ & 9 & $h^{\star}$ & $q$ & $\mathbf{h}^{*}$ & $q$ & $h^{*}$ & $q$ & $\mathrm{~h}^{\star}$ & $q$ & \\
\hline 5 & 1 & 773 & 29 & 1741 & 65 & 2837 & 157 & 4013 & 221 & 5309 & 257 & 6373 & 181 & 7717 & 673 & 8893 & 173 \\
\hline 13 & 1 & 797 & 37 & 1789 & 13 & 2861 & 261 & 4021 & 361 & 5333 & 149 & 6389 & 197 & 7741 & 421 & 933 & 425 \\
\hline 29 & 1 & 821 & 17 & 1861 & 53 & 2909 & 145 & 4093 & 325 & 5381 & 181 & 6397 & 137 & 7757 & 401 & 8941 & 505 \\
\hline 37 & 1 & 829 & 145 & 1877 & 53 & 2917 & 61 & 4133 & 205 & 5413 & 481 & 6421 & 613 & 7789 & 289 & 9013 & 637 \\
\hline 53 & 1 & 853 & 17 & 1901 & 53 & 2957 & 85 & 4157 & 173 & 5437 & 369 & 6469 & 677 & 7829 & 405 & 9029 & 169 \\
\hline 61 & 1 & 877 & 37 & 1933 & 29 & 3037 & 61 & 4229 & 101 & 5477 & 197 & 6581 & 225 & 7853 & 173 & 9109 & 277 \\
\hline 101 & 5 & 941 & 41 & 1949 & 125 & 3061 & 65 & 4253 & 181 & 5501 & 149 & 6637 & 305 & 7877 & 145 & 9133 & 625 \\
\hline 109 & 17 & 997 & 25 & 1973 & 45 & 3109 & 117 & 4261 & 53 & 5557 & 481 & 6653 & 229 & 7901 & 149 & 9157 & 245 \\
\hline 149 & 9 & 1013 & 25 & 1997 & 85 & 3181 & 185 & 4349 & 325 & 5573 & 269 & 6661 & 1165 & 7933 & 245 & 9173 & 317 \\
\hline 57 & 5 & 1021 & 41 & 2029 & 169 & 3221 & 85 & 4357 & 85 & 5581 & 53 & 6701 & 145 & 7949 & 125 & 9181 & 169 \\
\hline 173 & 5 & 1061 & 73 & 2053 & 41 & 3229 & 641 & 4373 & 185 & 5653 & 153 & 6709 & 125 & 8053 & 541 & 9221 & 197 \\
\hline 181 & 25 & 1069 & 29 & 2069 & 89 & 3253 & 229 & 4397 & 261 & 5669 & 85 & 6733 & 625 & 8069 & 157 & 9277 & 549 \\
\hline 197 & 5 & 1093 & 185 & 2141 & 61 & 3301 & 49 & 4421 & 85 & 5693 & 229 & 6781 & 401 & 8093 & 425 & 9293 & 289 \\
\hline 229 & 17 & 1109 & 9 & 2213 & 8 & 3373 & 241 & 4493 & 197 & 5701 & 505 & $\begin{array}{l}6829 \\
6869\end{array}$ & $\begin{array}{l}265 \\
233\end{array}$ & 101 & 369 & 341 & 257 \\
\hline 39 & 13 & 117 & 85 & 2221 & 101 & 3389 & 125 & 4517 & 145 & 5717 & 261 & & 425 & 8117 & 221 & 9349 & 185 \\
\hline 277 & 17 & 1181 & 37 & 2237 & 97 & 3413 & 153 & 4549 & 65 & 5741 & 233 & 6949 & 325 & 8221 & 365 & 9397 & 169 \\
\hline 293 & 9 & 1213 & 157 & 2269 & 125 & 34 & 15 & 4597 & 89 & 5749 & 1105 & 69 & 01 & 37 & 173 & 13 & 405 \\
\hline 016 & 13 & 229 & 20 & 22 & 29 & 3469 & 229 & 46 & 145 & 5813 & 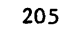 & 7013 & 325 & 8269 & 481 & 9421 & 577 \\
\hline 349 & 5 & 1237 & 125 & 2309 & 109 & 3517 & 73 & 4637 & 145 & 5821 & 305 & 7069 & 433 & 8293 & 225 & 9437 & 261 \\
\hline 373 & 5 & 1277 & 89 & 2333 & 85 & 3533 & 117 & 4733 & 225 & 5861 & 265 & 7109 & 369 & 8317 & 173 & 9461 & 441 \\
\hline 389 & 41 & 1301 & 25 & 2341 & 73 & 35 & 65 & 4789 & 81 & 5869 & 293 & 7213 & 157 & 8389 & 313 & 9533 & 377 \\
\hline 397 & 13 & 1373 & 45 & 2357 & 121 & 557 & 121 & 4813 & 145 & 5981 & 1005 & 7229 & 233 & 429 & 397 & 9613 & 641 \\
\hline 421 & 25 & 1381 & 153 & 2381 & 37 & 3581 & 289 & 4861 & 801 & 6029 & 313 & 7237 & 909 & 8461 & 365 & 9629 & 221 \\
\hline 461 & 25 & 1429 & 41 & 2389 & 25 & 3613 & 125 & 4877 & 193 & 6037 & 113 & 7253 & 265 & 8501 & 241 & 9661 & 1105 \\
\hline 09 & 13 & 453 & 221 & 2437 & 53 & 3637 & 405 & 4909 & 325 & 6053 & 109 & 7309 & 89 & 8573 & 261 & 9677 & 221 \\
\hline 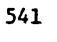 & 61 & 1493 & 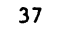 & 7רט & 85 & 3677 & 121 & 4933 & 389 & 6101 & 305 & 7333 & 425 & 8581 & 85 & 9733 & 2 \\
\hline 557 & 13 & 1549 & 25 & 2549 & 149 & 3701 & 145 & 4957 & 245 & 6133 & 137 & 7349 & 305 & 8597 & 521 & 9749 & 205 \\
\hline 513 & 25 & 1597 & 89 & 2557 & 205 & 3709 & 145 & 4973 & 137 & 6173 & 233 & 7477 & 865 & 8629 & 477 & 9781 & 493 \\
\hline 53 & 25 & 1613 & 45 & 2621 & 145 & 3733 & 365 & 5021 & 365 & 6197 & 325 & 7517 & 317 & 8669 & 293 & 9829 & 625 \\
\hline 61 & 9 & 1621 & 233 & 2677 & 361 & 3797 & 125 & 5077 & 125 & 6221 & 305 & 7541 & 725 & 8677 & 657 & 9901 & 1429 \\
\hline 577 & 25 & 1637 & 85 & 2693 & 89 & 3821 & 365 & 5101 & 565 & 6229 & 281 & 7549 & 85 & 8693 & 401 & 9941 & 22 \\
\hline 701 & 25 & 1669 & 85 & 2741 & 85 & 3853 & 245 & 5189 & 261 & 6269 & 257 & 7573 & 841 & 8741 & 585 & 9949 & 377 \\
\hline 709 & 61 & 1693 & 53 & 2749 & 85 & 3877 & 73 & 5197 & 117 & 6277 & 125 & 7589 & 225 & 8821 & 449 & 9973 & 1009 \\
\hline סני & 45 & 1709 & o & . & 101 & 4018 & J & 5237 & 120 & 6301 & 857 & 7621 & 485 & 8837 & 205 & & \\
\hline 757 & 125 & 1733 & 81 & 2797 & 289 & 3989 & 113 & 5261 & 185 & 6317 & 233 & 7669 & 1583 & 8861 & 169 & & \\
\hline
\end{tabular}


TABLE 2

Coset sums and numbers of quartic residues in subintervals of $q$

\begin{tabular}{|c|c|c|c|c|c|c|c|c|c|c|}
\hline$q$ & $\mathrm{~s}_{0}$ & $s_{1}$ & $\mathrm{~s}_{2}$ & $\mathrm{~s}_{3}$ & $\mathrm{~N}_{0}$ & $\mathrm{~N}_{1}$ & $\mathrm{~N}_{2}$ & $\mathrm{~N}_{3}$ & $\mathrm{~N}_{0}+\mathrm{N}_{3}$ & $N_{1}+N_{2}$ \\
\hline 13 & 1 & 1 & 2 & 2 & 2 & 0 & 1 & 0 & 2 & 1 \\
\hline 29 & 4 & 3 & 3 & 4 & 2 & 0 & 2 & 3 & 5 & 2 \\
\hline 37 & 4 & 5 & 5 & 4 & 3 & 3 & 1 & 2 & 5 & 4 \\
\hline 53 & 7 & 7 & 6 & 6 & 3 & 3 & 2 & 5 & 8 & 5 \\
\hline 61 & 7 & 8 & 8 & 7 & 5 & 4 & 2 & 4 & 9 & 6 \\
\hline 101 & 14 & 12 & 11 & 13 & 6 & 3 & 6 & 10 & 16 & 9 \\
\hline 109 & 11 & 12 & 16 & 15 & 12 & 5 & 7 & 3 & 15 & 12 \\
\hline 149 & 17 & 17 & 20 & 20 & 14 & 6 & 9 & 8 & 22 & 15 \\
\hline 157 & 19 & 18 & 20 & 21 & 12 & 7 & 11 & 9 & 21 & 18 \\
\hline 173 & 23 & 22 & 20 & 21 & 10 & 9 & 9 & 15 & 25 & 18 \\
\hline 181 & 19 & 22 & 26 & 23 & 18 & 11 & 9 & 7 & 25 & 20 \\
\hline 197 & 25 & 26 & 24 & 23 & 12 & 13 & 9 & 15 & 27 & 22 \\
\hline 229 & 27 & 26 & 30 & 31 & 19 & 10 & 16 & 12 & 31 & 26 \\
\hline 269 & 31 & 34 & 36 & 33 & 23 & 16 & 12 & 16 & 39 & 28 \\
\hline 277 & 36 & 32 & 33 & 37 & 17 & 12 & 21 & 19 & 36 & 33 \\
\hline 293 & 35 & 38 & 38 & 35 & 22 & 19 & 13 & 19 & 41 & 32 \\
\hline 317 & 39 & 37 & 40 & 42 & 23 & 15 & 22 & 19 & 42 & 37 \\
\hline 349 & 45 & 44 & 42 & 43 & 21 & 20 & 20 & 26 & 47 & 40 \\
\hline 373 & 45 & 46 & 48 & 47 & 27 & 22 & 22 & 22 & 49 & 44 \\
\hline 389 & 44 & 48 & 53 & 49 & 34 & 23 & 20 & 20 & 54 & 43 \\
\hline 397 & 49 & 52 & 50 & 47 & 25 & 28 & 20 & 26 & 51 & 48 \\
\hline 421 & 52 & 49 & 53 & 56 & 30 & 20 & 30 & 25 & 55 & 50 \\
\hline 461 & 58 & 61 & 57 & 54 & 30 & 30 & 20 & 35 & 65 & 50 \\
\hline 509 & 64 & 61 & 63 & 66 & 36 & 24 & 32 & 35 & 71 & 56 \\
\hline 541 & 62 & 68 & 73 & 67 & 43 & 36 & 29 & 27 & 70 & 65 \\
\hline 557 & 70 & 72 & 69 & 67 & 35 & 36 & 29 & 39 & 74 & 65 \\
\hline 613 & 73 & 76 & 80 & 77 & 45 & 38 & 36 & 34 & 79 & 74 \\
\hline 653 & 79 & 84 & 84 & 79 & 45 & 44 & 34 & 40 & 85 & 78 \\
\hline 661 & 81 & 84 & 84 & 81 & 45 & 42 & 36 & 42 & 87 & 78 \\
\hline 677 & 87 & 87 & 82 & 82 & 41 & 41 & 36 & 51 & 92 & 77 \\
\hline
\end{tabular}

Acknowledgement. I am grateful to Duncan A. Buell for making available extremely helpful computer data and additionally indebted to Kenneth S. Williams for advice and assistance in preparing this paper.

\section{REFERENCES}

[1] Adrian Albert, The integers of normal quartic fields, Annals of Math., 31 (1930), 381-418.

[2] Bruce Berndt, Classical theorems on quadratic residues, Enseignement Math., 22 (1976), 261-304.

[3] Bruce C. Berndt and Ronald J. Evans, Sums of Gauss, Jacobi, and Jacobsthal, J. Number Theory, 11 (1979), 349-398. 
[4] Duncan A. Buell and Richard H. Hudson, Solutions of certain quaternary quadratic systems, Pacific J. Math. , 114 (1984), 23-45.

[5] A. Cauchy, Mém. Institut de France, 17 (1840), 697; Oeuvres, (1), III, 388, Comptes Réndus, Paris 10 (1840), 451.

[6] L. E. Dickson, Cyclotomy and trinomial congruences, Trans. Amer. Math. Soc., 37 (1935), 363-380.

[7] P. G. Lejeune Dirichlet, Recherches sur diverses applications de l'analyse infinitésimale à la théorie des nombres, J. Reine Angew Math., 281 (1840), 134-155.

[8] Hugh Edgar and Brian Peterson, Some contributions to the theory of cyclic quartic extensions of the rationals, J. Number Theory, 12 (1980), 77-83.

[9] Helmut Hasse, Über die Klassenzahl abelscher Zahlkorper Akademie-Verlag, Berlin, 1952.

[10] Richard H. Hudson, Kenneth S. Williams, and Duncan A. Buell, Extension of a Theorem of Cauchy and Jacobi, submitted for publication.

[11] Richard H. Hudson and Kenneth S. Williams, A class number formula for certain quartic fields, Carleton Mathematical Series, No. 174, 1981, Carleton University, Ottawa, Canada.

[12] Emma Lehmer, On Euler's criterion, J. Austral. Math. Soc., 1 (1959), 64-70.

[13] Joseph B. Muskat and Yun-Cheng Zee, On the uniqueness of solutions of certain Diophantine equations, Proc. Amer. Math. Soc., 49 (1975), 13-19.

[14] W. Narkiewicz, Elementary and Analytic Theory of Algebraic Numbers, Polish Scientific Publishers, Warsaw, 1974.

[15] Ivan Niven and Herbert S. Zuckerman, An Introduction to the Theory of Numbers, John Wiley and Sons Inc., New York, 1972.

[16] Bennett Setzer, The determination of all imaginary, quartic, Abelian number fields with class number 1, Math. Comp., 35 (1980), 1383-1386.

[17] Albert Leon Whiteman, Theorems analogous to Jacobsthal's theorem, Duke Math., 16 (1949), 619-626.

Received February 16, 1983.

University of South Carolina

COLUMbia, SC 29208 


\title{
PACIFIC JOURNAL OF MATHEMATICS \\ EDITORS
}

\author{
DoNALD BABBITT (Managing Editor) \\ University of California \\ Los Angeles, CA 90024 \\ J. DugunduI \\ University of Southern California \\ Los Angeles, CA 90089-1113 \\ R. FINN \\ Stanford University \\ Stanford, CA 94305 \\ HERMANN FlasChKa \\ University of Arizona \\ Tucson, AZ 85721
}

C. C. Moore

University of California

Berkeley, CA 94720

ARTHUR OGUS

University of California

Berkeley, CA 94720

Hugo Rossi

University of Utah

Salt Lake City, UT 84112

H. SAMELSON

Stanford University

Stanford, CA 94305

ASSOCIATE EDITORS
R. ARENS
E. F. BECKENBACH
B. H. NeUMANN
F. WOLF
K. YoSHIDA (1906-1982)

\section{SUPPORTING INSTITUTIONS}

UNIVERSITY OF ARIZONA
UNIVERSITY OF BRITISH COLUMBIA
CALIFORNIA INSTITUTE OF TECHNOLOGY
UNIVERSITY OF CALIFORNIA
MONTANA STATE UNIVERSITY
UNIVERSITY OF NEVADA, RENO
NEW MEXICO STATE UNIVERSITY
OREGON STATE UNIVERSITY
UNIVERSITY OF ARIZONA
CALIFORNIA INSTITUTE OF TECHNOLOGY
UNIVERSITY OF CALIFORNIA
UNIVERSITY OF NEVADA, RENO
OREGON STATE UNIVERSITY
UNIVERSITY OF OREGON
UNIVERSITY OF SOUTHERN CALIFORNIA
STANFORD UNIVERSITY
UNIVERSITY OF HAWAII
UNIVERSITY OF TOKYO
UNIVERSITY OF UTAH
WASHINGTON STATE UNIVERSITY
UNIVERSITY OF WASHINGTON 


\section{Pacific Journal of Mathematics}

Vol. 115, No. $1 \quad$ September, 1984

Carlos Andradas Heranz and José Manuel Gamboa Mutuberría, A note on projections of real algebraic varieties $\ldots \ldots \ldots \ldots \ldots \ldots \ldots \ldots \ldots \ldots$

Jürgen Appell and Maria Patrizia Pera, Noncompactness principles in nonlinear operator approximation theory $\ldots \ldots \ldots \ldots \ldots \ldots \ldots \ldots \ldots \ldots$

Timothy John Carlson, Extending Lebesgue measure by infinitely many

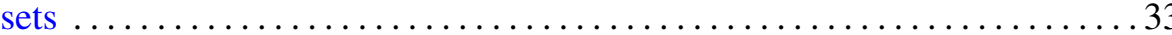

Donald S. Coram and Paul Frazier Duvall, Jr., Non-cell-like

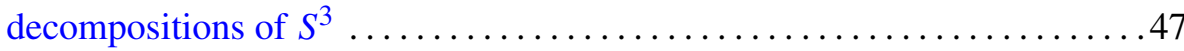

Edward Norman Dancer, Order intervals of selfadjoint linear operators and

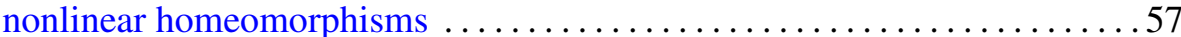

Ralph Jay De Laubenfels, Well-behaved derivations on $C[0,1] \ldots \ldots \ldots 73$

D. Feyel and A. de La Pradelle, Sur certaines extensions du théorème

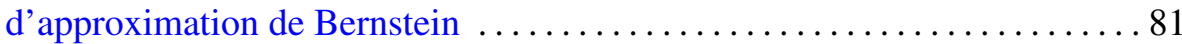

Colin C. Graham and Bertram Manuel Schreiber, Bimeasure algebras on

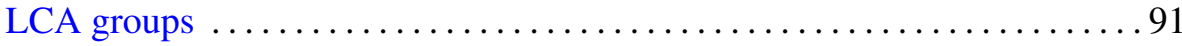

Richard Howard Hudson, Class numbers of imaginary cyclic quartic fields

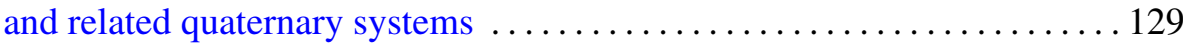

Carl Groos Jockusch, Jr. and Iraj Kalantari, Recursively enumerable sets and van der Waerden's theorem on arithmetic progressions . . . . . . . . 143

J. F. McClendon, On noncontractible valued multifunctions ........... 155 Akihiko Miyachi, Weak factorization of distributions in $H^{p}$ spaces $\ldots \ldots \ldots 165$ Ezzat S. Noussair and Charles Andrew Swanson, Global positive solutions of semilinear elliptic problems

Jon Christopher Snader, Strongly analytic subspaces and strongly

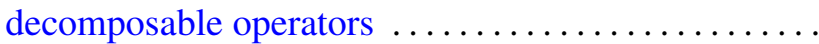

Boguslaw Tomaszewski, A construction of inner maps preserving the Haar measure on spheres

Akihito Uchiyama, The Fefferman-Stein decomposition of smooth functions and its application to $H^{p}\left(\mathbf{R}^{n}\right) \ldots \ldots \ldots$ 